University of Nebraska - Lincoln

DigitalCommons@University of Nebraska - Lincoln

$11-17-2007$

\title{
Characterization of Polymeric Proteins from Vitreous and Floury Sorghum Endosperm
}

B. loerger

S. R. Bean

M. R. Tuinstra

Jeffrey F. Pedersen

University of Nebraska-Lincoln, jpedersen1@unl.edu

J. Erpelding

See next page for additional authors

Follow this and additional works at: https://digitalcommons.unl.edu/usdaarsfacpub

Part of the Agricultural Science Commons

loerger, B.; Bean, S. R.; Tuinstra, M. R.; Pedersen, Jeffrey F.; Erpelding, J.; Lee, K. M.; and Herrman, T. J., "Characterization of Polymeric Proteins from Vitreous and Floury Sorghum Endosperm" (2007).

Publications from USDA-ARS / UNL Faculty. 212.

https://digitalcommons.unl.edu/usdaarsfacpub/212

This Article is brought to you for free and open access by the U.S. Department of Agriculture: Agricultural Research Service, Lincoln, Nebraska at DigitalCommons@University of Nebraska - Lincoln. It has been accepted for inclusion in Publications from USDA-ARS / UNL Faculty by an authorized administrator of DigitalCommons@University of Nebraska - Lincoln. 


\section{Authors}

B. loerger, S. R. Bean, M. R. Tuinstra, Jeffrey F. Pedersen, J. Erpelding, K. M. Lee, and T. J. Herrman 


\title{
Characterization of Polymeric Proteins from Vitreous and Floury Sorghum Endosperm
}

\author{
B. Ioerger ${ }^{\dagger}$ S. R. Bean, ${ }^{*}{ }^{\dagger}$ M. R. Tuinstra, ${ }^{\dagger}$ J. F. Pedersen, ${ }^{\S}$ J. Erpelding, \\ K. M. LEE ${ }^{\perp}$ AND T. J. HERRMAN ${ }^{\perp}$ \\ USDA-ARS, Manhattan, Kansas 66502, Department of Agronomy, Kansas State University, \\ Manhattan, Kansas 66506, USDA-ARS, Lincoln, Nebraska 68583, USDA-ARS Tropical Agriculture \\ Research Station, 2200 Pedro Albizu Campos Avenue, Suite 201, Mayaguez, Puerto Rico, and Texas \\ A\&M University, College Station, Texas 77483
}

\begin{abstract}
Differences in protein content and composition between vitreous and floury endosperm were investigated using a number of different techniques. Differences in protein cross-linking between vitreous and floury endosperm were investigated using differential solubility, size exclusion chromatography (SEC), and analysis of sulfhydryl content and composition. Vitreous endosperm was found to have higher levels of total protein and kafirins, but floury endosperm had a higher proportion of $\gamma$-kafirins than the vitreous. Floury endosperm was found to have higher levels of SDS-soluble proteins than SDS-insoluble proteins extracted using sonication than vitreous endosperm. Conversely, vitreous endosperm had a greater proportion of the insoluble proteins. SEC analysis of the polymeric proteins revealed that the insoluble proteins had more polymeric proteins than did the soluble proteins, indicating greater cross-linking and a larger $M_{\mathrm{w}}$ distribution. Vitreous endosperm was also found to have a greater percentage (i.e., a higher ratio of disulfide to total sulfhydryls) of disulfide bonds than floury endosperm. These results show that the proteins in vitreous endosperm have a higher degree of cross-linking and a greater $M_{w}$ distribution than those found in floury endosperm.
\end{abstract}

KEYWORDS: Sorghum; cereal; protein; kernel hardness; cross-linking; kafirin

\section{INTRODUCTION}

Grain hardness is an important grain quality attribute that plays a role in the processing of cereal grains and in the enduse quality of cereal grain based products such as breads and snack foods $(1,2)$. Grain hardness also plays a role in plant defense against molds and from insect attack (3). Therefore, grain hardness is an important economic and end-use quality trait in cereal grains.

Endosperm hardness in maize and sorghum has been positively correlated with both protein content and protein composition (3-9). The most abundant endosperm proteins of maize and sorghum are the prolamins $(10,11)$, which have been divided into subclasses based on solubility, structure, and amino acid sequence (12). The $\alpha$ subclass comprises the majority of the kafirins and makes up between $60 \%$ and $70 \%$ of total protein $(10,11)$. The $\alpha$-prolamins are located primarily in the interior of protein bodies while the $\beta$ - and $\gamma$-prolamins are present on the outer edges of the protein bodies $(3,5,7)$.

* Corresponding author. Tel: 785-776-2725. Fax: 785-537-5534. E-mail: scott.bean@gmprc.ksu.edu.

${ }^{\dagger}$ USDA-ARS, Manhattan.

* Kansas State University.

$\S$ USDA-ARS, Lincoln.

"USDA-ARS Tropical Agriculture Research Station.

${ }^{\perp}$ Texas A\&M University.
Past research has associated specific subclasses of kafirin proteins with grain hardness in sorghum and maize. The majority of publications in this area have reported that the vitreous endosperm of these grains is higher in total protein and total prolamin, while the floury endosperm is richer in $\gamma$-prolamins compared to vitreous endosperm (3). Chandrashekar and Mazhar (3) described the relationship between the prolamin subclasses and grain hardness as follows: "The $\gamma$-prolamins form the cement while the $\alpha$-prolamins are the bricks." These authors also postulated that both the content and distribution of $\alpha$ - and $\gamma$-kafirins (sorghum prolamins) were responsible for modifying endosperm texture, with the $\alpha$-kafirin responsible for protein body size and the $\gamma$-kafirin conferring rigidity by cross-linking the outer edges of the protein bodies (7). Furthermore, these authors reported that for a kernel to be hard the protein bodies needed to be large (high levels of kafirin) with strong crosslinking (high levels of $\gamma$-kafirin).

The above studies and most studies in general on sorghum proteins have all focused on the kafirin subclass composition. This is typically done by extracting the proteins under reducing conditions and analyzing the extracts via SDS-PAGE or RPHPLC. However, recently the polymeric proteins of sorghum were studied using techniques similar to those used in the study of the large gluten protein polymers found in wheat (13) and 
Table 1. Grain Traits and Hardness Indices for Sorghum Samples Used

\begin{tabular}{|c|c|c|c|c|c|c|}
\hline ATx623 $\times$ RTx430 & 15.9 & 99.5 & 24.4 & 2.2 & 84.0 & 9.3 \\
\hline Pioneer 82G63 & 12.5 & 71.2 & 29.8 & 2.3 & 79.0 & 8.3 \\
\hline A8PR1059 × LG35 & 15.7 & 85.2 & 26.1 & 2.4 & 82.9 & 9.7 \\
\hline Wheatland $\times \mathrm{KS} 115$ & 15.2 & 55.4 & 47.9 & 2.8 & 75.6 & 11.5 \\
\hline Wheatland $\times$ KS115 & 14.1 & 55.3 & 41.3 & 2.6 & 74.2 & 8.8 \\
\hline
\end{tabular}

\footnotetext{
${ }^{a}$ Abrasive hardness index. ${ }^{b}$ Single kernel characterization system (SKCS) hardness index. ${ }^{c}$ Determined using the SKCS.
}

traditional SDS-PAGE (14). The polymeric proteins of maize have also been characterized using similar techniques (15).

Considerable work on the differences in protein composition of vitreous and floury endosperm has been done. However, this research has focused mainly on the kafirin subclasses and not the polymeric proteins, even though evidence points in the direction of protein cross-linking as an important part of grain hardness in sorghum (3). This project addresses the question of the role of cross-linking of sorghum proteins into larger polymeric groups in the role of grain hardness by using a number of protein analytical techniques to study the protein composition (in terms of both reduced proteins and unreduced polymeric proteins) of isolated vitreous and floury endosperm.

\section{MATERIALS AND METHODS}

Samples. Eight sorghum samples were selected from a large collection of samples held at the USDA-ARS GMPRC laboratory and were selected to span a range of hardness values. All of the sorghum samples were grown in Kansas except for B94C174, a waxy sorghum, which was grown in Nebraska. Two of the samples were the same hybrid, Wheatland $\times \mathrm{KS} 115$, grown in two different locations in Kansas.

Sample Preparation. Sorghum kernels (10 g) were decorticated using the tangential abrasive decortication device (TADD) as described previously $(16,17)$. Samples were decorticated for various times $(1-4$ min), and kernels were stained according to the method of Scheuring and Rooney (18). Stained kernels were visually inspected to determine the optimum decortication time for removal of the bran.

Decorticated samples were degermed and cut in half with a scalpel, and the floury endosperm was removed with a dremel motor tool (Dremel, Racine, WI) and collected. Hard endosperm was then ground in a coffee grinder for $10 \mathrm{~s}$ followed by grinding in a mortar and pestle and then sieved through a no. 40 mesh screen. Floury endosperm was recovered as a fine powder during the kernel drilling procedure, and thus no grinding was necessary.

Protein Extraction. Total kafirins were extracted from ground (25 $\mathrm{mg}$ ) whole grain and endosperm fractions as described in Bean et al. (19) and analyzed by reversed-phase (RP) HPLC and free zone capillary electrophoresis (FZCE). For analysis of polymeric proteins, a multistep procedure was used. Soluble proteins (SP) were first extracted from $10 \mathrm{mg}$ of ground endosperm using $0.5 \mathrm{~mL}$ of a $12.5 \mathrm{mM}$ sodium borate, $\mathrm{pH} 10$, buffer with $2 \%$ SDS (w/v) for 30 min with continual vortexing (no reducing agent in buffer). After being centrifuged, the supernatant was removed, and the insoluble proteins (IP) were extracted from the residue using sonication ( $30 \mathrm{~s}$ at $10 \mathrm{~W}$ in the above $\mathrm{pH} 10$ buffer). After centrifugation, the residue proteins (RP) were extracted from the remaining pellet under reducing conditions using the $\mathrm{pH} 10$ sodium borate/SDS buffer with $2 \% \beta$-mercaptoethanol (v/v) added. Aliquots of each extract (SP, IP, and RP) were removed and analyzed by size exclusion high-performance liquid chromatography (SEC). The percentages of SP, IP, and RP in a given sample were determined by summing the SEC peak areas across each extract (SP area + IP area + RP area $=$ total area) and then dividing each individual extract area by the total area (e.g., SP area/total area). To characterize the composition of each extract (i.e., SP, IP, and RP), individual peaks in the SEC chromatograms were integrated.

To further characterize the proteins in each of these extracts, an additional aliquot was taken from each extract, and $\beta$-mercaptoethanol was added to a final concentration of $2 \%(\mathrm{v} / \mathrm{v})$. Samples were then allowed to incubate for $30 \mathrm{~min}$ at room temperature and then analyzed by RP-HPLC. To evaluate the amount of protein extracted by the multistep extraction procedure, three of the sorghum hybrids were selected that spanned the range of SCKS hardness values. The above extraction procedure was carried out on a larger scale on whole endosperm (100 mg sample to $1 \mathrm{~mL}$ solvent), and the amount of protein remaining after all extracts was measured using nitrogen combustion.

Protein Analysis. All HPLC separations were carried out using an Agilent 1100 HPLC system. RP-HPLC conditions were as described in Bean et al. (19). Proteins were separated by SE-HPLC using a Biosep-3000 column (Phenomenx, Torrance, CA) with a $50 \mathrm{mM}$ sodium phosphate, $\mathrm{pH} 7.0$, buffer containing $1 \%$ SDS as mobile phase with a flow rate of $1 \mathrm{~mL} / \mathrm{min}$ as described in Bean et al. (20). HPCE was carried out as described in Bean et al. (19) using a Beckman PACE 2100 instrument.

Nitrogen Combustion. Protein content was measured using nitrogen combustion via a Leco FP-528 nitrogen determinator (St. Joseph, MI) according to AACC method 46-30 (crude protein-combustion method) (21). Nitrogen was converted to protein using a factor of 6.25 .

Grain Hardness Measures. The single kernel characterization system (SKCS) was used to obtain grain hardness, kernel weight, and diameter $(17,22)$. The abrasive hardness index (AHI) was measured using the tangential abrasive dehulling device (TADD) (16).

Sulfhydryl Measurements. Free sulfhydryl content $(\mathrm{SH})$ was determined using the methods of Thannhauser et al. (23) and Chan and Wasserman (24) as described in detail in Lee et al. (25).

Statistical Analysis. All data were plotted, and statistical analysis was conducted using Microsoft Excel and Microcal Origin.

\section{RESULTS}

Physical Grain Attributes. There was a large range of grain hardness among the samples tested as measured by both the SKCS and the TADD (Table 1). As expected from samples varying in hardness, the other physical properties of the grains also varied among the samples. Kernel weight, for example, varied almost 2-fold (Table 1). Correlations among the physical properties were as expected with kernel weight highly correlated to kernel diameter $(r=0.92, p<0.05$; data not shown) and kernel diameter negatively correlated to the percent vitreousity ( $r=-0.75, p<0.05$; data not shown). SKCS hardness values and AHI were not significantly correlated to each other in this study.

Protein and Kafirin Content and Composition. Total protein and kafirin content for vitreous and floury endosperm is shown in Table 2. As expected from previous studies, vitreous endosperm had greater levels of total protein than the floury endosperm and had much higher amounts of kafirins (both absolute and on a percent basis). The total $\gamma$-kafirin peak area 
Table 2. Kafirin Composition of Endosperm Fractions Averaged across All Samples

\begin{tabular}{|c|c|c|}
\hline \multirow[b]{2}{*}{ measurement } & \multicolumn{2}{|c|}{ endosperm fraction ${ }^{f}$} \\
\hline & vitreous & floury \\
\hline total protein ${ }^{a}$ & $9.8 a$ & $7.5 b$ \\
\hline$\gamma$-kafirin area $^{b}$ & $1829 a$ & $999 \mathrm{~b}$ \\
\hline$\alpha / \beta$-kafirin area ${ }^{c}$ & $31612 a$ & $8144 b$ \\
\hline$\gamma$-kafirin $(\%)^{d}$ & $5.4 a$ & $11.2 b$ \\
\hline$\alpha / \beta$-kafirin $(\%)^{e}$ & $94.1 \mathrm{a}$ & $88.8 b$ \\
\hline
\end{tabular}

${ }^{a}$ Total protein $\%$ of endosperm fractions as determined by nitrogen combustion. ${ }^{b}$ RP-HPLC peak area of $\gamma$-kafirins. ${ }^{c}$ RP-HPLC peak area of $\alpha$ - and $\beta$-kafirins. $d \%$ RP-HPLC area of $\gamma$-kafirins [ $(\gamma$ peak area $) /(\gamma$ peak area $+\alpha$ and $\beta$ peak area)]. ${ }^{e} \%$ RP-HPLC area of $\alpha$ - and $\beta$-kafirins [( $\alpha$ and $\beta$ peak area $) /(\gamma$ peak area $+\alpha$ and $\beta$ peak area)]. ${ }^{f}$ Values followed by the same letter in the same row are not significantly different $(p<0.05)$.
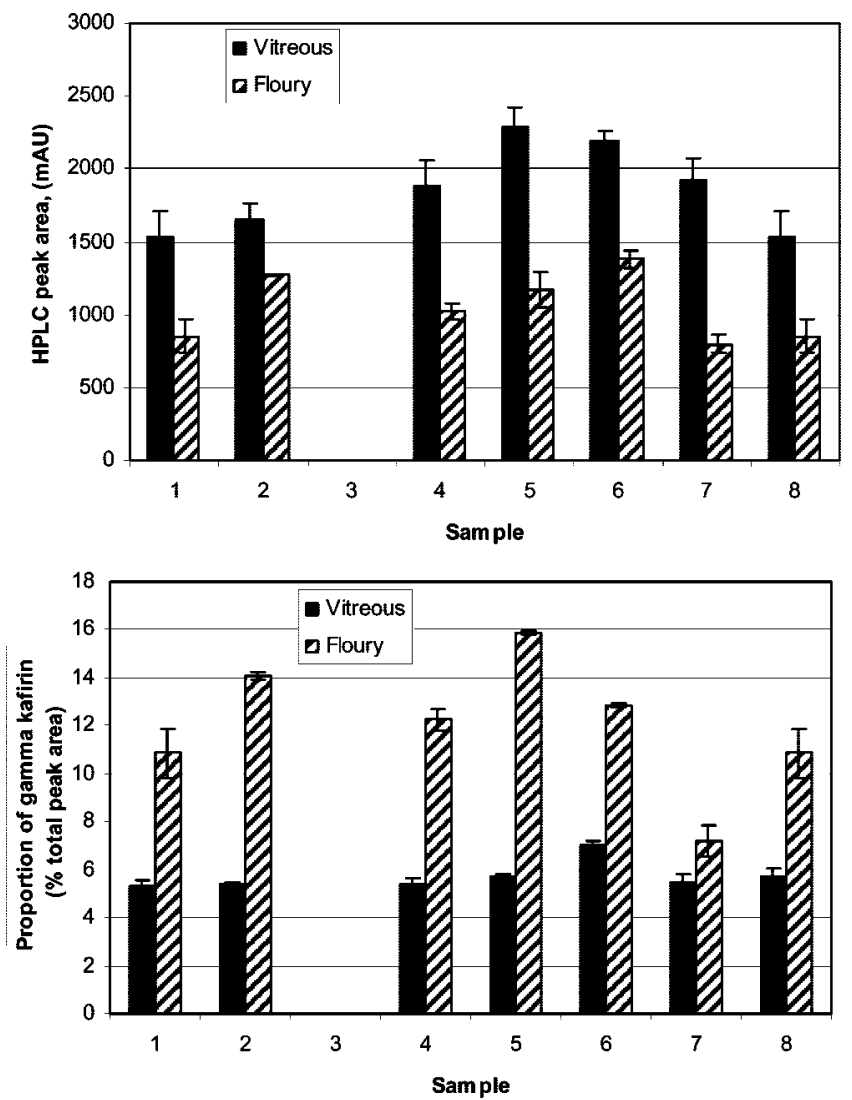

Figure 1. $\gamma$-Kafirin HPLC peak area and $\% \gamma$-kafirin peak area (of total HPLC area) for individual sorghum samples. Note no data are shown for sample 3 due to lack of available sample for these analyses. Sample numbers correspond to the order the samples are listed in Table 1. Error bars represent standard deviation $(n=2)$.

and the percent $\gamma$-kafirin $(\gamma$-kafirin peak area/total peak area) in the vitreous and floury endosperm as determined by RPHPLC for the individual samples are shown in Figure 1. The percent of $\gamma$-kafirin in both the vitreous and floury endosperm varied among the samples, but in all cases the floury endosperm contained a higher percentage of $\gamma$-kafirins than did the vitreous endosperm.

Kafirins extracted from vitreous and floury endosperm were analyzed by RP-HPLC and FZCE to determine the overall kafirin composition in each type of endosperm (selected example shown in Figure 2). For all samples, kafirin separations were similar to those shown in Figure 2, and visual inspection of the RP-HPLC and FZCE kafirin patterns showed that qualitatively the kafirins were essentially identical between the two
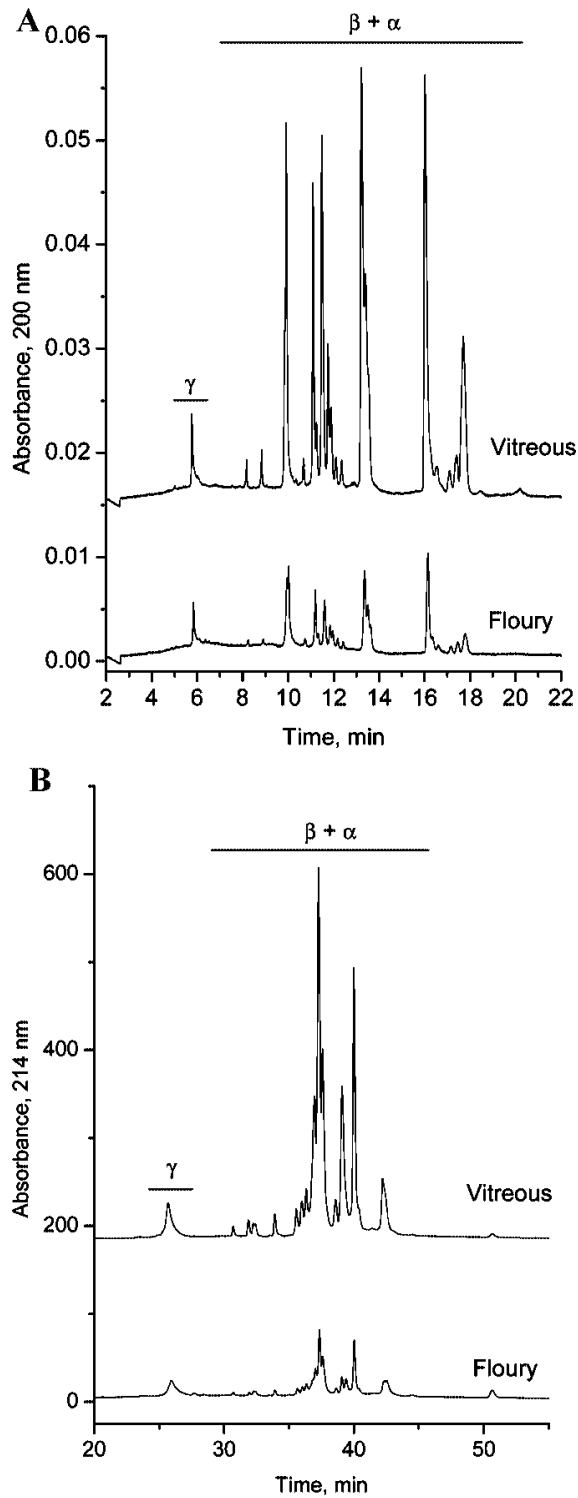

Figure 2. (A) FZCE and (B) RP-HPLC separations of total kafirins extracted from vitreous and floury endosperm from the sorghum hybrid Mycogen X00ML337.

endosperm fractions. Quantitatively, however, the kafirins extracted from floury endosperm showed large differences when compared to the kafirins from the vitreous endosperm. The floury endosperm obviously had lower levels of kafirins. The floury endosperm also appeared to have lower levels of the peak in the $\alpha$ region of the FZCE separations just before 18 min (Figure 2). A similar difference was seen in the $\alpha / \beta$ range in the RP-HPLC separations at $\sim 43 \mathrm{~min}$ in the RP-HPLC separations (Figure 2). While the absolute $\gamma$-kafirin peak content was greater in the vitreous endosperm fractions, the proportion of $\gamma$-kafirins to the $\alpha / \beta$-kafirins in the floury endosperm was greater than in the vitreous endosperm, with $\gamma$-kafirins in the floury endosperm making up almost twice the percentage of kafirins as in the vitreous endosperm (Table 2), with only one sample (Mycogen X00ML337) deviating substantially from this trend (Figure 1).

Sulfhydryl Content and Composition. Free sulfhydryl (F$\mathrm{SH})$, total sulfhydryl (T-SH), and disulfide (S-S) contents were measured on vitreous and floury endosperm samples in addition to protein characterization. When comparing averages between the vitreous and floury endosperm across all samples, vitreous endosperm had lower levels of both F-SH and T-SH (on a per 
Table 3. Sulfhydryl Content and Composition of Vitreous and Floury Endosperm Averaged across All Samples

\begin{tabular}{|c|c|c|}
\hline \multirow[b]{2}{*}{ measurement } & \multicolumn{2}{|c|}{ endosperm fraction ${ }^{e}$} \\
\hline & vitreous & floury \\
\hline free $\mathrm{SH}(\mathrm{F}-\mathrm{SH})^{a}$ & $12.7 a$ & $68.6 b$ \\
\hline total SH $(\mathrm{T}-\mathrm{SH})^{b}$ & $59.2 a$ & $139.2 b$ \\
\hline disulfide content $(\mathrm{S}-\mathrm{S})^{c}$ & $23.3 a$ & $35.3 b$ \\
\hline ratio S-S/T-SH ${ }^{d}$ & $0.39 a$ & $0.25 b$ \\
\hline
\end{tabular}

${ }^{a}$ Free sulfhydryl content, $\mathrm{nmol} / \mathrm{mg}$ of protein. ${ }^{b}$ Total sulfhydryl content, $\mathrm{nmol} /$ $\mathrm{mg}$ of protein. ${ }^{c}$ Disulfide content, $\mathrm{nmol} / \mathrm{mg}$ of protein. ${ }^{d}$ Ratio of disulfide content to total sulfhydryl content. ${ }^{e}$ Values followed by the same letter in the same row are not significantly different $(p<0.05)$.

protein basis) than did floury endosperm (Table 3). Disulfide content was also lower in vitreous endosperm than in floury, though the difference was not as great as for the F-SH and T-SH measurements (Table 3). The ratio of S-S to T-SH was greater in the vitreous endosperm than in the floury endosperm, indicating more disulfide cross-links (i.e., a greater percent of the T-SH were in the form of S-S) in the vitreous endosperm relative to the floury endosperm (Table 3). When looking at the data from the individual samples, the same trends as for the averages for the endosperm types were seen (Figure 3). In each sample, free $\mathrm{SH}$ and total $\mathrm{SH}$ were much higher in the floury endosperm compared to the vitreous endosperm. For the disulfide bonds, differences between vitreous and floury endosperm were readily apparent except for two samples (A8PR1059xLG35 and Mycogen X00ML337) which did not share similar overall grain traits (Table 1). Likewise, the ratio of disulfide to total $\mathrm{SH}$ bonds was greater for the vitreous endosperm than the floury in all samples, the same as when the averages of vitreous and floury were compared.

Polymeric Proteins. To characterize the polymeric proteins in vitreous and floury endosperm, a sequential extraction scheme was used which divided proteins into SDS "soluble" proteins, insoluble proteins (extracted using ultrasound), and residue proteins (extracted with reducing agent). Data for these solubility classes for each of the sorghum samples are shown in Figure 4. Floury endosperm either contained the same or higher amounts of SP than did vitreous endosperm among the samples tested (Figure 4A). Vitreous endosperm contained higher amounts of IP than did floury endosperm, and in the majority of samples (Figure 4B), RP was similar between vitreous and floury endosperm among the samples (Figure 4C). Comparisons of the averages for these solubility classes across all samples for both vitreous and floury endosperm are shown in Table 4. On average, the vitreous endosperm had lower levels of the soluble proteins than did floury endosperm, though as mentioned above, this difference was greater in some samples than in others. Conversely, the vitreous endosperm showed higher levels of insoluble proteins than did the floury endosperm. Levels of residue protein were not significantly different between the two types of endosperm (Table 4).

To test the amount of total protein extracted with the above extraction scheme, three samples varying in SKCS hardness values were extracted, and the protein in the residue was determined by nitrogen combustion. This test revealed that 95-97\% of total protein was extracted (data not shown).

To characterize the polymeric protein composition of the SP, IP, and RP extracts, samples were analyzed by SEC and the chromatograms divided into five peak regions (Figure 2). On the basis of the elution times of standard $M_{\mathrm{W}}$ marker proteins and by analyzing SEC patterns following reduction, peaks 1 and 2 were judged to contain mostly polymeric proteins while
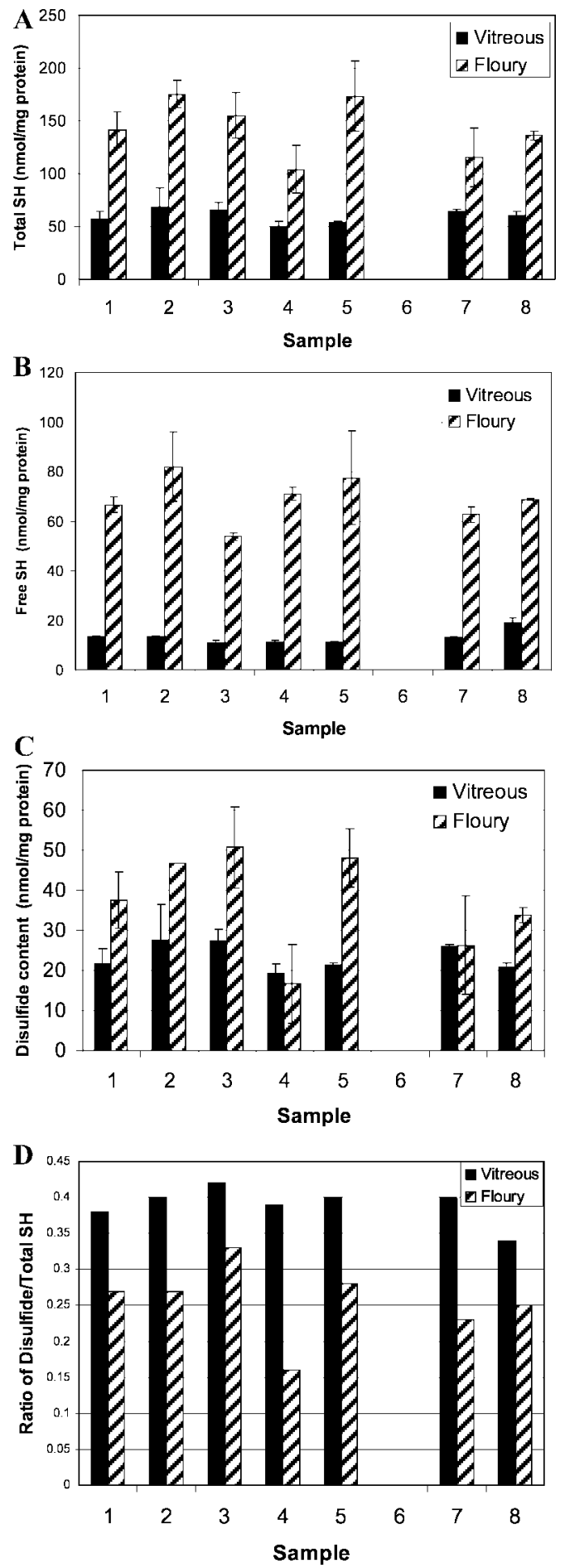

Figure 3. (A) Free $\mathrm{SH}$ bonds for vitreous and floury endosperm for individual samples, $\mathrm{nmol} / \mathrm{mg}$ of protein, (B) total $\mathrm{SH}$ bonds and free $\mathrm{SH}$ bonds for vitreous and floury endosperm for individual samples, $\mathrm{nmol} / \mathrm{mg}$ of protein, (C) disulfide (S-S) bonds for vitreous and floury endosperm for individual samples, nmol/mg of protein, and (D) ratio of disulfide to total SH for vitreous and floury endosperm for individual samples. Sample numbers correspond to the order in which the samples are listed in Table 1. Data are not shown for sample 6 due to the limited amount of sample available. Error bars represent standard deviation $(n=2)$.

peaks 3-5 contain monomeric proteins, which was in agreement with the results of Bean et al. (20) and El Nour et al. (13). The results of the SEC analysis are shown in Figure 3. In the vitreous endosperm, the SP extract was mainly peaks 3, 4, and 5 , which totaled over $70 \%$ of the total SEC area. In the IP 

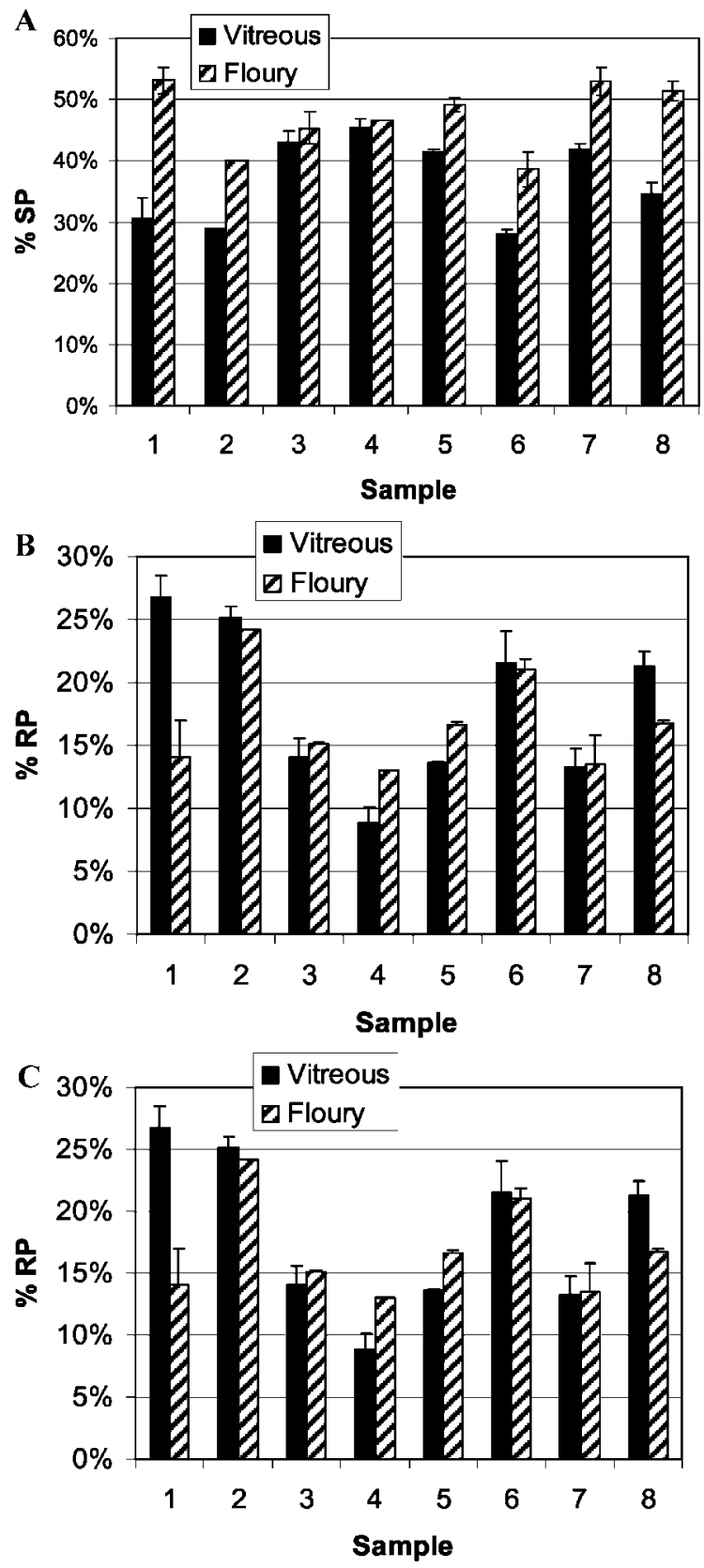

Figure 4. Percentage of (A) SDS-soluble proteins (SP), (B) SDS-insoluble proteins $(\mathrm{IP})$, and $(\mathrm{C})$ residue proteins $(\mathrm{RP})$ in individual sorghum samples. Sample numbers correspond to the order in which the samples are listed in Table 1. Error bars represent standard deviation $(n=2)$.

Table 4. Amount of Soluble, Insoluble, and Residue Protein in Vitreous and Floury Endosperm Averaged across All Samples

\begin{tabular}{ccc}
\hline & \multicolumn{2}{c}{ SEC peak area $(\%)^{d}$} \\
\cline { 2 - 3 } extraction & vitreous & floury \\
\hline $\mathrm{SP}^{a}$ & $36.7 \mathrm{a}$ & $47.2 \mathrm{~b}$ \\
$\mathrm{IP}^{b}$ & $45.3 \mathrm{a}$ & $35.9 \mathrm{~b}$ \\
$\mathrm{RP}^{c}$ & $18.0 \mathrm{a}$ & $16.9 \mathrm{a}$
\end{tabular}

${ }^{a}$ Soluble protein. ${ }^{b}$ Insoluble protein. ${ }^{c}$ Residue protein. ${ }^{d}$ Values followed by the same letter in the same row are not significantly different $(p<0.05)$.

extract, however, the percentage of peak 1 was greatly increased and was roughly equal to that of peaks 3 and 4 . In the floury SP extract, peaks 3 and 4 dominated, totaling almost $75 \%$ of the total SEC peak area. In the floury IP extract, the proportion of peak 1 greatly increased to make up almost $40 \%$ of the total
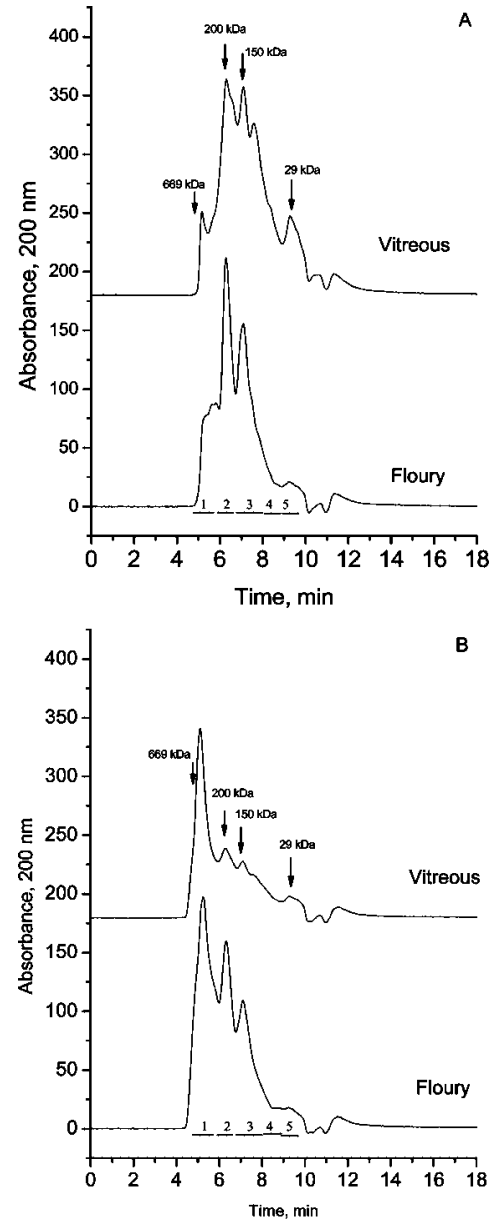

Figure 5. SEC separation of (A) SDS-soluble proteins (SP) and (B) SDSinsoluble proteins (IP) from vitreous and flour endosperm from the sorghum hybrid Mycogen X00ML337. Arrows indicate the approximate elution position of $M_{\mathrm{w}}$ markers, and numbered bars indicate the location of integrated peaks.

SEC area. In both the vitreous and floury RP extracts, peak 4 was the major peak (Table 4).

To further characterize the proteins present in the SP, IP, and $\mathrm{RP}$ extracts from the vitreous and floury endosperm, aliquots of each extract from two sorghum samples were lyophilized and then resuspended $\mathrm{pH} 10 \mathrm{SDS}$ buffer with $2 \% \beta$-ME added and the reduced proteins analyzed by RP-HPLC. The SP, IP, and RP from vitreous endosperm showed little difference in their chromatograms and resembled the chromatograms of a total kafirin extract (data not shown). However, in the floury endosperm fractions, the SP contain large amounts of peaks eluting in the $\gamma$-kafirin region, and the IP chromatograms showed reduced levels of peaks eluting in the $\gamma$-kafirin region (data not shown).

\section{DISCUSSION}

Previous research on prolamins and grain hardness in sorghum and maize has produced a number of ideas on how proteins influence kernel hardness. Most postulate that cross-linking of the $\gamma$-and possibly $\beta$-kafirins on the outer edges of the protein bodies plays a role. This cross-linking could be either to matrix protein, which is tightly compressed against protein bodies in the vitreous endosperm, or to other prolamins $(3,15,27,28)$.

While several hypotheses about protein cross-linking and grain hardness have been put forward, to date no studies have attempted to look directly at the polymeric proteins in isolated 
A
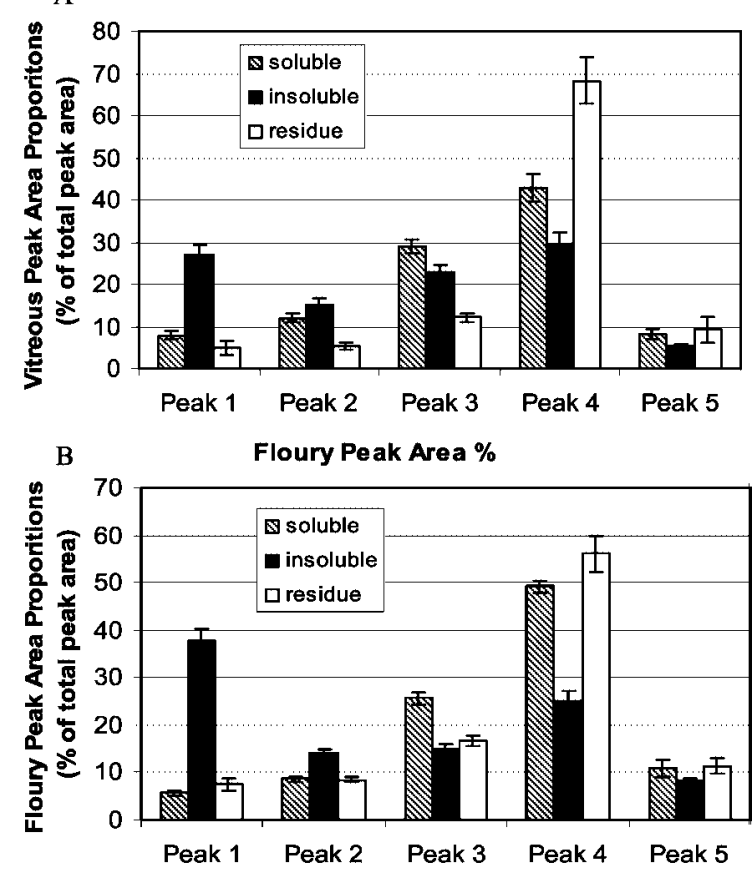

Figure 6. \% SEC peak area (of total peak area) for (A) vitreous endosperm and (B) floury endosperm. Error bars represent standard deviation $(n=2)$.

vitreous and floury endosperm. If protein cross-linking between kafirins and matrix protein, or simply within the matrix protein itself, varies between vitreous and floury endosperm, the analysis of unreduced protein extracts from the endosperm fractions should show such differences. To directly gain information on the polymeric protein content and composition of vitreous and floury endosperm, a multistep extraction procedure was used to divide sorghum proteins into SDS-soluble, SDS-insoluble, and residue proteins. This is often done in wheat as a method to determine the molecular weight distribution of the polymeric glutenin proteins (29) as the insoluble proteins are hypothesized to have a larger $M_{\mathrm{w}}$ distribution than the soluble proteins (and hence their insolubility), which was recently verified using laser light scattering (30). Thus, we applied the idea of differential solubility, frequently used to study the molecular weight distribution in wheat proteins, to study the cross-linked proteins of sorghum.

On the basis of the above, we hypothesized that if the protein cross-linking was different between vitreous and floury endosperm, the distribution of SP and IP would also differ between vitreous and floury endosperm. The results of the multistep extraction procedure showed that the floury endosperm had significantly more SP than did the vitreous endosperm (Table 4). Conversely, the vitreous endosperm had a higher percentage of the IP fraction than the floury endosperm. Following the wheat protein model, this would suggest that the vitreous endosperm had more of the larger (and therefore insoluble) polymeric proteins than did the floury endosperm. However, other factors such as increased hydrophobocity could also account for lack of solubility of the IP in sorghum. Complete characterization of the SP and IP using multiangle laser light scattering and mass spectrometry is currently underway.

Early classification schemes for sorghum proteins often utilized an aqueous alcohol solvent to extract a fraction called "kafirin 1". A second aqueous alcohol extract with a reducing agent was then used to extract a fraction called "kafirin 2". A similar scheme in the Landry and Moureaux produced extracted fractions labeled "II" and "III", respectively (31). The kafirin 1 and fraction II in these earlier papers would be roughly analogous to the SP extract in the current paper. Likewise, kafirin 2 and fraction III would be roughly equal to IP in the current study. Similar relationships of kafirin 1 and kafirin 2 to soluble and insoluble extracts were made by El Nour et al. (13). Using these earlier methods, vitreous endosperm was found to have higher levels of kafirin 2 than did floury endosperm during kernel development (6).

To further investigate the $M_{\mathrm{w}}$ distribution of the proteins in the vitreous and floury endosperm, the SP and IP extracts were analyzed by SEC (Figure 2). Note that in this sense the $M_{\mathrm{w}}$ distribution is referred to not in the ranges of the $M_{\mathrm{w}}$ of the proteins present but rather in the distribution of the amounts of proteins within the $M_{\mathrm{w}}$ ranges present. This is widely done in wheat proteins where sonication is needed to extract the largest insoluble proteins and which alters the original $M_{\mathrm{w}}$ ranges of the proteins, the largest of which elute in the void volume of the SEC columns in any case, making it difficult to judge the true range of $M_{\mathrm{w}}$ 's present (32). The use of nonreduced extractions followed by analysis of the proteins by SEC allowed for a direct look at the molecular weight distribution of these extracts, something not done previously (in all previous studies proteins have been reduced prior to analysis). Significant differences in the peak compositions were found between the vitreous and floury endosperm (Figure 3). In the SP extract, there were no major differences between the vitreous and floury endosperm except that floury endosperm had much higher levels of peak 4. This would seem to make sense if the solubility is related to the $M_{\mathrm{w}}$ distribution; i.e., the extraction process would be expected to extract similar material in both endosperm fractions. In the vitreous endosperm, IP had more of peak 1 material than peak 2, suggesting a shifted $M_{\mathrm{w}}$ distribution toward larger polymeric proteins. Overall levels of the polymeric peaks (1 and 2) were higher than in the SP, again showing a shift toward more polymeric proteins in IP than SP. The same trend was observed in the floury endosperm, though the lower $M_{\mathrm{w}}$ peaks 3 and 4 were not as prevalent relative to the polymeric proteins (1 and 2).

It was interesting that the floury IP had a high percentage of its protein in peak 1 than did the vitreous IP, which again indicates differences in cross-linking between the endosperm types. Taken together, this seems to indicate that there is a shift toward more polymeric proteins in the IP than SP and that the IP in the floury endosperm has more polymeric proteins relative to monomeric proteins. This could reflect a better extraction of protein in the floury endosperm; however, overall amounts of protein extracted by this procedure were similar between vitreous and floury endosperm, and the RP protein content was similar between the vitreous and floury endosperm. It was also interesting to note that the kafirin composition of the SP, IP, and RP from the vitreous endosperm did not vary much while the SP from the floury endosperm was highly enriched with $\gamma$-kafirins. As noted above, RP levels were similar between both vitreous and floury endosperm. These proteins were not extracted with either sonication or in the presence of reducing agents, suggesting that their solubility was not limited due to the $M_{\mathrm{w}}$, as both of these techniques would have reduced their $M_{\mathrm{w}}$. It is most likely that these are non-prolamin proteins, and similar results have been found in wheat (33).

In addition to the above protein studies, the sulfhydryl content and composition of the vitreous and floury endosperm was evaluated. Floury endosperm had higher levels of both F-SH and T-SH on a per protein basis. This could be due to the higher 
proportion of $\gamma$-kafirins in the floury endosperm, which are known to be high in Cys (34). However, in the vitreous endosperm the ratio of $\mathrm{S}-\mathrm{S}$ to $\mathrm{T}-\mathrm{SH}$ was greater; thus a greater proportion of the T-SH was involved in disulfide bonds, indicating greater cross-linking of proteins in the vitreous endosperm compared to the floury endosperm.

The results of this paper indicate that vitreous endosperm had a greater level of protein cross-linking than did floury endosperm and that this cross-linking produced a larger $M_{w}$ distribution than found in the floury endosperm. Vitreous endosperm has a much more compact structure with a continuous matrix than floury endosperm and therefore more opportunity for cross-linking between protein components (5). This was the opposite for the floury endosperm, where protein bodies were not tightly packed and the protein matrix was discontinuous and, thus, less opportunity for protein cross-linking (5).

Several relationships were found between the various protein classes measured in this study that may help to explain the crosslinking of sorghum proteins. For instance, the amount of $\gamma$-kafirin in vitreous endosperm was negatively correlated to the amount of SP in vitreous endosperm $(r=-0.85, p<0.5$, $n=6$; data not shown). Interestingly, the amount of $\gamma$-kafirin in vitreous endosperm was positively correlated to the amount of RP in vitreous endosperm $(r=0.92, p<0.05, n=6$; data not shown). These correlations are based on a limited number of samples and should be regarded as preliminary; however, they do seem to indicate that the amount of $\gamma$-kafirin in the vitreous endosperm is related to the distribution of the solubility classes and, thus, the $M_{\mathrm{w}}$ distribution. The RP may be the most difficult to extract due to the most cross-linking of the proteins through the $\gamma$-kafirins. It remains to be seen if RP proteins have an even larger $M_{\mathrm{w}}$ than the IP or if they are the most difficult to extract due to some other properties of being highly crosslinked. No significant relationships between kafirin content and composition to SP, IP, or RP in the floury endosperm were found. Likewise, neither the total kafirins in vitreous endosperm or floury endosperm was correlated to their respective SP, IP, or RP levels. Thus, the $\gamma$-kafirins in the vitreous endosperm seem to have the most obvious relationships to indicators of protein cross-linking in the vitreous endosperm. The fact that, in the floury endosperm, the SP extract was enriched with $\gamma$-kafirins may suggest that whatever cross-links the $\gamma$-kafirins in the vitreous endosperm do not cross-link them in the floury endosperm, or perhaps in the floury endosperm they are crosslinked to themselves. El Nour et al. (13) also reported that $\gamma$-kafirins were important in the cross-linking of kafirins along with $\beta$-kafirins when analyzing samples extracted from whole grain; however, further study on the composition of sorghum polymeric proteins in sorghum is needed to confirm the roles and mechanisms of $\gamma$ - and $\beta$-kafirins in protein cross-linking. It is also important to note that, in studying the polymeric protein composition of sorghum, vitreous and floury endosperm need to be studied separately.

\section{LITERATURE CITED}

(1) Bettge, A. D.; Morris, C. F. Relationships among grain hardness, pentosan fractions, and end-use quality of wheat. Cereal Chem. 2000, 77, 241-247.

(2) Cagampang, G. B.; Kirleis, A. W. Relationship of sorghum grain hardness to selected physical and chemical measurements of grain quality. Cereal Chem. 1984, 61, 100-105.

(3) Chandrashekar, A.; Mazhar, H. The biochemical basis and implications of grain strength in sorghum and maize. J. Cereal Sci. 1999, 30, 193-207.
(4) Wall, J. S.; Bietz, J. A. Differences in corn endosperm proteins in developing seeds of normal and opaque- 2 corn. Cereal Chem. 1987, 64, 275-280.

(5) Shull, J. M.; Chandrashekar, A.; Kirleis, W.; Ejeta, G. Development of sorghum endosperm in varieties of varying hardness. Food Struct. 1990, 9, 253-267.

(6) Mazhar, H.; Chandrashekar, A. Differences in kafirin composition during endosperm development and germination in sorghum cultivars of varying hardness. J. Cereal Sci. 1993, 70, 667-671.

(7) Mazhar, H.; Chandrashekar, A. Quantification and distribution of kafirins in the kernels of sorghum cultivars varying in endosperm hardness. J. Cereal Sci. 1995, 21, 155-162.

(8) Dombrink-Kurtzman, M. A.; Bietz, J. A. Zein composition in hard and soft endosperm of maize. Cereal Chem. 1997, 70, 105-108.

(9) Pratt, R. C.; Paulis, J. W.; Miller, K.; Nelsen, T.; Bietz, J. A. Association of zein classes with maize kernel hardness. Cereal Chem. 1995, 72, 162-167.

(10) Wallace, J. C.; Lopes, M. A.; Paiva, E.; Larkins, B. A. New methods for extraction and quantification of zeins reveal a high content of $\alpha$-zein in modified opaque- 2 maize. Plant Physiol. 1990, 92, 191-196.

(11) Hamaker, B. R.; Mohamed, A. A.; Habben, J. E.; Huang, C. P.; Larkins, B. A. Efficient procedure for extracting maize and sorghum kernel proteins reveals higher prolamin contents than the conventional method. Cereal Chem. 1995, 72, 583-588.

(12) Shull, J. M.; Watterson, J. J.; Kirleis, A. W. Proposed nomenclature for the alcohol proteins (kafirins) of Sorghum bicolor (L. Moench) based on molecular weight, solubility, and structure. $J$. Agric. Food Chem. 1991, 39, 83-87.

(13) El Nour, I. N. A.; Peruffo, A. D. B.; Curioni, A. Characterisation of sorghum kafirins in relation to their cross-linking behaviour. J. Cereal Sci. 1998, 28, 197-207.

(14) Nunes, A.; Correia, I.; Barros, A.; Delgadillo, I. Characterization of kafirin and zein oligomers by preparative sodium dodecyl sulfate-polyacrylamide gel electrophoresis. J. Agric. Food Chem. 2005, 53, 639-643.

(15) Wall, J. S.; Cooker, L. A.; Bietz, J. A. Structure and origin of maize endosperm alcohol-insoluble glutelin. J. Agric. Food Chem. 1988, 36, 722-728.

(16) Oomah, B. D.; Reichert, R. D.; Youngs, C. G. A novel, multisample, tangential abrasive dehulling device (TADD). Cereal Chem. 1981, 58, 392-395.

(17) Bean, S. R.; Chung, O. K.; Tuinstra, J. F.; Erpelding, J. Evaluation of the Single Kernel Characterization System (SKCS) for Measurement of Sorghum Grain Attributes. Cereal Chem. 2006, 83, 108-113.

(18) Scheuring, J. F.; Rooney, L. W. A staining procedure to determine the extent of bran removal in pearled sorghum. Cereal Chem. 1979, 56, 545-547.

(19) Bean, S. R.; Lookhart, G. L.; Bietz, J. A. Acetonitrile as a buffer additive for the separation of maize (Zea mays L.) and sorghum (Sorghum bicolor L.) storage proteins by HPCE. J. Agric. Food Chem. 2000, 48, 318-327.

(20) Bean, S. R.; Ioerger, B. P.; Park, S. H.; Singh, H. Interaction Between Sorghum Protein Extraction and Precipitation Conditions on Yield, Purity, and Composition of Purified Protein Fractions. Cereal Chem. 2006, 83, 99-107.

(21) AACC International. AACC Methods 46-30 and 76-31. Approved Methods of the American Association of Cereal Chemists, 10th ed.; AACC International: St. Paul, MN, 2000

(22) Pedersen, J. F.; Martin, C. R.; Felker, F. C.; Steele, J. L. Application of the single kernel wheat characterization technology to sorghum grain. Cereal Chem. 1996, 73, 421-423.

(23) Thannhauser, T. W.; Konishi, Y.; Scheraga, H. A. Analysis for disulfide bonds in peptides and proteins. Methods Enzymol. 1987, 143, 115-119.

(24) Chan, K. Y.; Wasserman, B. P. Direct colorimetric assay of free thiol groups and disulfide bonds in suspensions of solubilized and particulate cereal proteins. Cereal Chem. 1993, 70, 22-26.

(25) Lee, K.-M.; Bean, S. R.; Alavi, S.; Herrman, T. J.; Waniska, R. D. Physical and biochemical properties of maize hardness and 
extrudates of selected hybrids. J. Agric. Food Chem. 2006, 54, 4260-4269, 2006.

(26) Watterson, J. J.; Shull, J. M.; Kirleis, A. W. Quantitation of $\alpha-$, $\beta$-, and $\gamma$-kafirins in vitreous and opaque endosperm of Sorghum bicolor. Cereal Chem. 1993, 70, 452-457.

(27) Paiva, E.; Kris, A. L.; Peixoto, M. J. V. V. D.; Wallace, J. C.; Larkins, B. A. Quantitation and distribution of $\gamma$-zein in the endosperm of maize kernels. Cereal Chem. 1991, 68, 176-279.

(28) Robutti, J. L.; Borras, F. S.; Eyherabide, G. H. Zein compositions of mechanically separated coarse and fine portions of maize kernels. Cereal Chem. 1997, 74, 75-78.

(29) Southan, M.; MacRitchie, F. Molecular weight distribution of wheat proteins. Cereal Chem. 1999, 76, 827-836.

(30) Bean, S. R.; Lookhart, G. L. Factors influencing the characterization of gluten proteins by SEC-MALLS. Cereal Chem. 2001, 78, 608-618.

(31) Landry, J.; Moureaux, T. Heterogeniety of the glutelins of the grain of corn: Selective extraction and composition in amino acids of the insoluble fractions. Bull. Soc. Chem. Biol. 1970, 52, 10211037.

(32) Southan, M.; MacRitchie, F. Molecular weight distribution of wheat proteins. Cereal Chem. 1999, 76, 827-836.

(33) Sapirstein, H. D.; Fu, B. X. Intercultivar variation in the quantity of monomeric proteins, soluble and insoluble glutenin, and residue protein in wheat flour and relationships to breadmaking quality. Cereal Chem. 1998, 75, 500-507.

(34) Belton, P. S.; Delgadillo, I.; Halford, N. G.; Shewry, P. R. Kafirin structure and functionality. J. Cereal Sci. 2006, 44, 272-286.

Received for review June 8, 2007. Revised manuscript received October 10, 2007. Accepted October 14, 2007. Mention of firm names or trade products does not constitute endorsement by the U.S. Department of Agriculture over others not mentioned.

JF0716883 